\title{
Inter-Observer Agreement and Image Quality of Model- Based Algorithm Applied to the Coronary Artery Disease Reporting and Data System Score
}

\section{Davide Ippolito}

San Gerardo Hospital: Ospedale San Gerardo

Cammillo Talei Franzesi

San Gerardo Hospital: Ospedale San Gerardo

\section{Cecilia Cangiotti}

San Gerardo Hospital: Ospedale San Gerardo

\section{Luca Riva}

San Gerardo Hospital: Ospedale San Gerardo

\section{Andrea De Vito}

San Gerardo Hospital: Ospedale San Gerardo

\section{Davide Gandola}

San Gerardo Hospital: Ospedale San Gerardo

\section{Cesare Maino ( $\square$ mainocesare@gmail.com )}

San Gerardo Hospital: Ospedale San Gerardo https://orcid.org/0000-0002-5742-802X

\section{Sandro Sironi}

Papa Giovanni XXIII Hospital: Aziende Socio Sanitarie Territoriale Papa Giovanni XXIII

\section{Research Article}

Keywords: Coronary Artery Disease, Coronary Artery Disease - Reporting and Data System, Radiation Exposure, Cardiac Computed Tomography Angiography, Model-Based Iterative Reconstruction algorithm.

Posted Date: August 6th, 2021

DOI: https://doi.org/10.21203/rs.3.rs-775670/v1

License: (c) (i) This work is licensed under a Creative Commons Attribution 4.0 International License. Read Full License 


\section{Abstract}

Purpose: To evaluate the inter-observer agreement of the CAD-RADS reporting system and compare image quality between model-based iterative reconstruction algorithm (MBIR) and standard iterative reconstruction algorithm (IR) of low dose cardiac computed tomography angiography (CCTA).

Methods: One-hundred-sixty patients undergone a 256-slice MDCT scanner using low-dose CCTA combined with prospective ECG-gated techniques were prospectively enrolled. CCTA protocols were reconstructed with both MBIR and IR. Each study was evaluated by two readers using the CAD-RADS lexicon.

Vessels enhancement, image noise, SNR, and CNR were computed in the axial native images and inter-observer agreement was assessed. Radiation dose exposure as dose-length product (DLP) and effective dose (ED) were finally reported.

Results: The overall agreement was very good $(k=0.90)$. Moreover, a significantly higher value of subjective qualitative analysis, SNR, and CNR in MBIR images compared to IR were found, due to a lower noise level $(p<0.05)$. The mean DLP measured was $63.9 \mathrm{mGy}{ }^{*} \mathrm{~cm}$ and the mean effective dose was $0.9 \mathrm{mSv}$.

Conclusion: Inter-observer agreement of CAD-RADS was excellent confirming the importance, the feasibility, and the reproducibility of the CAD-RADS scoring system for CCTA. Moreover, lower noise and higher image quality with MBIR compared to IR were found.

\section{Introduction}

In the last years, computed tomography (CT) technology has shown essential changes and improvements, and cardiac CT angiography (CCTA) has rapidly evolved as the most non-invasive test in the detection of coronary artery disease (CAD) in low to intermediate-risk patients.

The progressive expansion, from 64 to 320 slices, allowed an increment of spatial and temporal resolution, leading to a more precise evaluation of atherosclerosis plaque composition (calcified, non-calcified, and mixed plaque). Moreover, CCTA can detect subclinical CAD, positive vessel remodeling, and spotty calcifications (1), in addition to the evaluation of extracardiac findings.

The 2016 updated National Institute for Health and Care Excellence (NICE) guidelines removed the pre-test probability model and evaluated the diagnostic accuracy of the non-invasive test against invasive coronary angiography (ICA) for significant stenosis detection, suggesting CCTA as the first-line test in CAD positive patients $(2,3)$. A recent meta-analysis of diagnostic accuracy of CCTA demonstrated an overall sensitivity of $99 \%$ and specificity of $87 \%$, compared to ICA as the reference standard, with an added high NPV (up to $100 \%)(4,5)$.

SCOT-HEART and PROMISE studies have also shown the usefulness of CCTA in addition to or as an alternative to functional testing, respectively $(6,7)$. However, it has been reported that post-CCTA patient management is often sub-optimal (8). Essentially the new European Society of Cardiology (ESC) guidelines give greater prominence to CCTA to confirm CAD, in particular in case of obstructive CAD not ruled out with clinical assessment, CCTA is equally recommended as an alternative initial approach.

In the last few years, given the large incidence of CAD and therefore of patients undergoing CCTA, a high level of expertise for image interpretation and standardization in the reports are required (9). The society of cardiovascular 
computed tomography (SCCT), the American college of radiology (ACR), and the North American society of cardiovascular imaging (NASCl) drew up the Coronary Artery Disease - Reporting and Data System (CAD-RADS) score, to help improving communication between radiologist and cardiologist and suggesting subsequent clinical management $(10,11)$.

In these settings, CCTA became a widespread diagnostic tool in CAD patients and, consequently, dose reduction should be mandatory according to the ALARA principle. Nowadays, this is possible due to the introduction in clinical practice of the new model-based iterative reconstruction (MBIR) algorithms, allowing to perform low-dose examinations, reducing image noise, and leading to equal or better diagnostic quality compared to standard-dose CT reconstructed with iterative reconstruction (IR) algorithm.

On this basis, this study aims to assess the inter-observer agreement of the CAD-RADS reporting system of low dose CCTA and compare the subjective and objective image quality between the MBIR algorithm with the IR one.

\section{Material And Methods}

\subsection{Study population}

This study was performed in a single university center and was approved by our institutional review board. Written informed consent was obtained from all patients.

All patients with clinical suspicion of CAD and after a cardiological evaluation were prospectively included.

Exclusion criteria were: 1) patients with unstable angina, 2) patients with severe renal failure (eGFR $<30$ $\mathrm{mL} / \mathrm{min} / 1.73 \mathrm{~m}^{2}$ ) or other contraindications for iodinated contrast material (i.e.previous allergic reaction), 3) heart rate $>85 \mathrm{bpm}$ with contraindications to the use of $\beta$-blocker, 4) presence of arrhythmia or atrial fibrillation, 5) unstable clinical condition, 6) inability to perform a breath-hold.

\subsection{CCTA protocol}

All patients underwent low-kV CCTA protocol combined with prospective ECG-gated technique by using a 256MDCT scanner (iCT Elite, Philips Medical Systems, Best, The Netherlands), a dual-mode scout (on the coronal and sagittal plane) to select the acquisition volume with the lowest scan length according to the patient's anatomy.

The $80 \mathrm{kV}$ setting increased to $100 \mathrm{kV}$ for patients with BMI over 30 , automated tube-current modulation, with a preset value depending on the patient's shape and geometry from the scout image, and a dose right index of 7 was used to reduce the total radiation dose. The other scan parameters were as follows: collimation $128 \times 0.625$; rotation time $0.27 \mathrm{sec}$; thickness $0.67 \mathrm{~mm}$; increment $0.335 \mathrm{~mm}$; FOV $250 \mathrm{~mm}$; matrix 512 × 512 .

CT data were acquired with step \& shoot acquisition with a prospective ECG-triggered axial acquisition, selecting the $78 \%( \pm 3 \%)$ of the cardiac cycle (R-R interval).

In each patient, an 18-gauge intravenous catheter was placed in an antecubital vein of the upper limb, and a contrast medium was injected using an automatic double-syringe injector (Medrad Stellant, Pittsburgh, PA, USA). We used a standardized dose of contrast medium volume of $70 \mathrm{ml}$ (lobidtritol 350 - Xenetix, Guerbet, Aulnay, France), with a flow rate of $4.5 \mathrm{~mL} / \mathrm{s}$ followed by saline flushing (volume $50 \mathrm{ml}$, flow rate $4.5 \mathrm{~mL} / \mathrm{s}$ ). 
If the baseline heart rate (HR) was $>65$ beats per minute (BPM) and patients had no contraindications for $\beta$ blockers, metoprolol (5-20 mg) was injected intravenously before the examination.

The start of scanning was obtained for each patient by using a bolus-tracking technique, with a trigger area manually placed at the proximal ascending aorta with $120 \mathrm{HU}$ as threshold and $8 \mathrm{~s}$ as a delay.

All raw data were reconstructed with the standard filter "Cardiac Routine" with both algorithms: MBIR (IMR, Level 1, Philips Healthcare, Cleveland, OH, USA) and IR (iDose ${ }^{4}$, Level 4, Philips Healthcare, Cleveland, OH, USA). CCTA protocol is summarized in Table 1.

Table 1

- Scanning parameters and reconstruction algorithms used

\begin{tabular}{|lll|}
\hline CT scan parameters & MBIR & IR \\
\hline Tube-voltage $(\mathrm{kV})$ & 80 & \\
\hline Tube-current $\left(\mathrm{mAs}{ }^{\star}\right)$ & Automated & \\
\hline Gantry rotation time $(\mathrm{s})$ & 0.27 & \\
\hline Detector configuration & $128 \times 0.625$ & \\
\hline FOV (mm) & 250 & $1.0 / 1.0$ \\
\hline Thickness; increment $(\mathrm{mm})$ & $0.67 / 0.34$ & $60 / 4.5$ \\
\hline CM volume (mL); flow rate $(\mathrm{mL} / \mathrm{s})$ & $60 / 4.5$ & 6 \\
\hline
\end{tabular}

\subsection{Image analysis}

Images were processed on a dedicated workstation (IntelliSpace Portal 9.0, Philips) to compute multiplanar reconstructions (MPR), maximum intensity projections (MIP), and volume rendering (VR) images. The CAD-RADS assessment categories and modifiers (10), quantitative and qualitative images analyses were performed by two radiologists with 4 (reader 1 ) and 7 years of experience (reader 2 ) in CCTA, CTA, and 3D vascular images interpretation, blinded each other and to clinical data.

\section{Qualitative image evaluation}

The diagnostic image quality of the ascending aorta and the coronary arteries (RCA, CTk, LAD, and LCX) was evaluated using a 5-point Likert scale for coronary CTA by the two readers, based on the presence of motion artifacts and image noise influencing subjective image quality, as follows: $5=$ excellent image quality, $4=$ good image quality, 3 = acceptable image quality, 2 = below-average image quality, 1 = poor image quality (13).

\section{Quantitative image evaluation}

Each study was evaluated using the CAD-RADS lexicon based on the degree of maximum coronary stenosis among vessel segments larger than $1.5 \mathrm{~mm}$ in diameter. All vessels were evaluated with a scoring system from 0 to 5 , as follows: $0=$ absence of atherosclerosis, $1=$ minimal stenosis or plaque with no stenosis $(1-24 \%), 2=$ mild stenosis (25-49\%), $3=$ moderate stenosis (50-69\%), 4A = severe stenosis $(60-79 \%)$ or $4 \mathrm{~B}=$ left main $>50 \%$ or 3 vessel obstructive (> 70\%), 5 = total occlusion (100\%). 
Moreover, CAD-RADS categories can be completed by modifiers as follows: $\mathrm{N}=$ non-diagnostic study, $\mathrm{S}=$ presence of a stent, $\mathrm{G}=$ presence of graft, and $\mathrm{V}=$ presence of vulnerable plaque (10).

We measured vessel contrast enhancement (mean attenuation value, $\mathrm{HU}$ ) and image noise, determined as the standard deviation of the attenuation values (SD) (Fig. 1), by manually placing a circular region of interest (ROI) in the center of the vascular lumen in ascending aorta $(A O)$, in the proximal segment of right coronary artery (RCA), common trunk (CTk), left anterior descending (LAD) and left circumflex (LCx).

We computed the signal-to-noise ratio (SNR) using the formula $\mathrm{SNR}=\frac{\mathrm{HU}_{\mathrm{A}}}{\mathrm{SD}_{\mathrm{A}}}$ where $\mathrm{HU}_{\mathrm{A}}$ is the mean attenuation of the coronary artery (at each proximal segment) and $\mathrm{SD}_{\mathrm{A}}$ is the standard deviation of the HU values. Finally, we also evaluated the contrast resolution by calculating the contrast-to-noise ratio (CNR) using the formula:

$\mathrm{CNR}=\frac{\mathrm{HU}_{\mathrm{A}}-\mathrm{HU}_{\mathrm{B}}}{\mathrm{SD}_{\mathrm{B}}}$

where $\mathrm{HU}_{\mathrm{A}}$ is the attenuation of the proximal tract of coronary arteries and the ascending aorta and $\mathrm{HU}_{\mathrm{B}}$ and $\mathrm{SD}_{\mathrm{B}}$ are the attenuation and standard deviation of the adjacent adipose tissue, as reported in the paper by C.H. Park et al (12).

Finally, for each patient examined with CCTA, we checked the presence of ICA as the reference standard, to confirm the diagnostic value.

\subsection{Radiation dose}

CT dose index (CTDIvol, $\mathrm{mGy}$ ) and CT dose-length product (DLP, $\mathrm{mGy} \cdot \mathrm{cm}$ ) were registered for all examinations. We also computed the effective dose (ED), using the formula ED $=k \times D L P$, where $k$ is the region-specific normalized effective dose (mSv/mGycm) derived from the paper by Deak et al (14). A k value of $0.0146 \mathrm{mSv} / \mathrm{mGy}-1 \cdot \mathrm{cm}-1(14)$ was currently used for estimating the effective dose from cardiovascular imaging procedures for adult patients.

\subsection{Statistical analysis}

Continuous variables were expressed as means and standard deviations and compared by using the MannWhitney test or t-Student's test, when appropriate.

The agreement between the two readers was assessed using the Cohen kappa or Weighted kappa coefficients (0.00-0.20 indicates slight agreement; 0.21-0.40, fair agreement; 0.41-0.60, moderate agreement; 0.61-0.80, substantial agreement; and 0.81-1.00, almost perfect agreement), in case of 2 or more than 2 categorical variables, respectively.

The comparison between the continuous variables measured by the two readers was assessed with Spearman correlation and the Bland-Altman Limits of Agreement (LOA) with the $95 \% \mathrm{Cls}$.

A p-value $<0.05$ was considered significant. The analysis was performed using SPSS software (v 26.0, SPSS Inc, Chicago, Illinois).

\section{Results}

\subsection{Study population}

A total of 160 patients $(M / F=49 / 31)$ with a mean age of $68 \pm 9$ years (range $33-78)$ were prospectively enrolled. 
All CCTA examinations were completed in a single breath-hold, without any complications and any adverse effect after $\mathrm{CM}$ injection.

\subsection{Image analysis}

Qualitative analysis

The inter-observer agreement of the subjective image quality evaluation was good $(k=0.630)$. Moreover, the overall image quality evaluation of the two reconstruction algorithms demonstrated a statistically higher score in MBIR images compared to IR, for both the two readers [reader 1: MBIR 4 (IQR: $3-4$ ) vs IR $3(2-3), p<0.000$; reader 2: MBIR 4 (3-4) vs IR $3(2-3), p<0.0001]$.

Quantitative analysis

The mean attenuation value of RCA, CTk, and LAD arteries in MBIR images was significantly higher compared to IR (Table 2). Also, the LCx artery and AO demonstrated a higher intra-vessel density value, without a statistically significant difference $(p=0.122$ and $p=0.445$, respectively). Moreover, the CNR and SNR were higher in MBIR compared to IR images, with a statistical difference $(p<0.0001)$, as shown in Table 2.

Table 2

Comparison between MBIR e IR for HU, SD (noise), CNR and SNR.

\begin{tabular}{|c|c|c|c|c|c|c|c|c|c|c|c|c|}
\hline \multirow[t]{2}{*}{ Vessels } & \multicolumn{3}{|c|}{$\mathrm{HU}( \pm \mathrm{SD})$} & \multicolumn{3}{|c|}{$S D( \pm S D)$} & \multicolumn{3}{|c|}{ CNR $( \pm$ SD) } & \multicolumn{3}{|c|}{ SNR ( \pm SD) } \\
\hline & MBIR & IR & $\begin{array}{l}\mathrm{p}- \\
\text { value }\end{array}$ & MBIR & IR & $\begin{array}{l}\mathrm{p}- \\
\text { value }\end{array}$ & MBIR & IR & $\begin{array}{l}\mathrm{p}- \\
\text { value }\end{array}$ & MBIR & IR & $\begin{array}{l}\mathrm{p}- \\
\text { value }\end{array}$ \\
\hline RCA & $\begin{array}{l}500 \\
\pm \\
123\end{array}$ & $\begin{array}{l}474 \\
\pm \\
126\end{array}$ & $\begin{array}{l}< \\
0.0001\end{array}$ & $\begin{array}{l}19 \pm \\
6\end{array}$ & $\begin{array}{l}26 \\
\pm 7\end{array}$ & $\begin{array}{l}< \\
0.0001\end{array}$ & $\begin{array}{l}28 \pm \\
13\end{array}$ & $\begin{array}{l}18 \\
\pm 8\end{array}$ & $\begin{array}{l}< \\
0.0001\end{array}$ & $\begin{array}{l}27 \pm \\
20\end{array}$ & $\begin{array}{l}13 \\
\pm 6\end{array}$ & $\begin{array}{l}< \\
0.0001\end{array}$ \\
\hline CTk & $\begin{array}{l}477 \\
\pm \\
119\end{array}$ & $\begin{array}{l}466 \\
\pm \\
131\end{array}$ & 0.032 & $\begin{array}{l}25 \pm \\
8\end{array}$ & $\begin{array}{l}29 \\
\pm \\
10\end{array}$ & $\begin{array}{l}< \\
0.0001\end{array}$ & $\begin{array}{l}26 \pm \\
12\end{array}$ & $\begin{array}{l}18 \\
\pm 8\end{array}$ & $\begin{array}{l}< \\
0.0001\end{array}$ & $\begin{array}{l}24 \pm \\
11\end{array}$ & $\begin{array}{l}12 \\
\pm 6\end{array}$ & $\begin{array}{l}< \\
0.0001\end{array}$ \\
\hline LAD & $\begin{array}{l}506 \\
\pm \\
119\end{array}$ & $\begin{array}{l}489 \\
\pm \\
132\end{array}$ & 0.034 & $\begin{array}{l}18 \pm \\
8\end{array}$ & $\begin{array}{l}27 \\
\pm 9\end{array}$ & $\begin{array}{l}<.0001 \\
\end{array}$ & $\begin{array}{l}29 \pm \\
13\end{array}$ & $\begin{array}{l}19 \\
\pm 9\end{array}$ & $\begin{array}{l}<.0001 \\
0.00\end{array}$ & $\begin{array}{l}29 \pm \\
18\end{array}$ & $\begin{array}{l}13 \\
\pm 9\end{array}$ & $\begin{array}{l}< \\
0.0001\end{array}$ \\
\hline LCx & $\begin{array}{l}505 \\
\pm \\
119\end{array}$ & $\begin{array}{l}490 \\
\pm \\
143\end{array}$ & 0.122 & $\begin{array}{l}18 \pm \\
7\end{array}$ & $\begin{array}{l}26 \\
\pm 9\end{array}$ & $\begin{array}{l}<.0001 \\
0.00\end{array}$ & $\begin{array}{l}28 \pm \\
14\end{array}$ & $\begin{array}{l}19 \\
\pm 8\end{array}$ & $\begin{array}{l}< \\
0.0001\end{array}$ & $\begin{array}{l}25 \pm \\
13\end{array}$ & $\begin{array}{l}13 \\
\pm 7\end{array}$ & $\begin{array}{l}<.0001 \\
0 .\end{array}$ \\
\hline$A O$ & $\begin{array}{l}480 \\
\pm \\
113\end{array}$ & $\begin{array}{l}481 \\
\pm \\
121\end{array}$ & 0.445 & $\begin{array}{l}25 \pm \\
8\end{array}$ & $\begin{array}{l}30 \\
\pm \\
11\end{array}$ & 0.110 & $\begin{array}{l}27 \pm \\
12\end{array}$ & $\begin{array}{l}18 \\
\pm 8\end{array}$ & $\begin{array}{l}< \\
0.0001\end{array}$ & $\begin{array}{l}28 \pm \\
14\end{array}$ & $\begin{array}{l}13 \\
\pm 7\end{array}$ & $\begin{array}{l}< \\
0.0001\end{array}$ \\
\hline
\end{tabular}


Table 3

CAD-RADS assessment categories between the 2 readers: the interobserver agreement was almost perfect $(\mathrm{k}=0.900)$.

\begin{tabular}{|lll|}
\hline CAD-RADS & Reader 1 & Reader 2 \\
\hline $\mathbf{0}$ & $34(21.25 \%)$ & $40(25 \%)$ \\
\hline $\mathbf{2}$ & $26(16.25 \%)$ & $20(12.5 \%)$ \\
\hline $\mathbf{3}$ & $22(13.75 \%)$ & $14(8.75 \%)$ \\
\hline 4A & $10(6.25 \%)$ & $20(12.5 \%)$ \\
\hline 4B & $16(10 \%)$ & $22(13.75 \%)$ \\
\hline $\mathbf{5}$ & $10(6.25 \%)$ & $4(2.5 \%)$ \\
\hline $\mathbf{N}$ & $24(15 \%)$ & $22(13.75 \%)$ \\
\hline G & $18(11.25 \%)$ & $18(11.25 \%)$ \\
\hline S & $10(6.25 \%)$ & $10(6.25 \%)$ \\
\hline N = non diagnostic study, S = presence of stent, G = presence of graft \\
\hline
\end{tabular}

Image noise was significantly lower in MBIR images compared with IR ones, in all vascular districts (all $p<0.05)$, except for the aorta $(p=0.110)$ (Table 2$)$.

The comparison of inter-reader evaluation of attenuation values and image noise didn't show any bias, as reported in the Bland-Altman plot (Fig. 2).

\section{CAD-RADS evaluation and comparison with invasive coronary angiography}

A very good inter-observer agreement for all CAD-RADS categories was found between the two readers $(k=0.900)$ and for modifiers $(k=0.920)$ (Table 3 ).

Fifty-eight (36.2\%) patients were classified as CAD-RADS 4 (A or B) and 10 (6.25) as CAD-RADS 5, and consequently advised to undergo invasive coronary angiography: the CAD-RADS category was confirmed in 63 examinations (93\%). For the other 5 patients (7\%) the CAD-RADS category was downgraded from 4A to 3 . All data regarding CAD-RADS categories are reported in Table 3.

\subsection{Radiation dose}

A mean DLP of $63.9 \pm 32.50 \mathrm{mGy}{ }^{\star} \mathrm{cm}$, mean CTDI of $4.32 \pm 1.40 \mathrm{mGy}$, and a mean ED of $0.9 \pm 0.47 \mathrm{mSv}$ were reported.

\section{Discussion}

This study aims to confirm the feasibility and reproducibility of CAD-RADS and improve reporting consistency. Our results demonstrated an excellent inter-observer agreement for CAD-RADS assessment categories and modifiers, in line with the previous report from Maroules et al (19): both early career reader and expert reader have proved a high concordance in CAD-RADS assessment, despite no experience in this scoring system. Szilveszter et al, using a 
reporting platform that determines automatically the CAD-RADS category (20), have achieved results slightly superior to our study: this difference could be explained by the fact that it was the first time that we used the CADRADS lexicon. In our series, the major difference in terms of the inter-observer agreement has been registered in our results for CAD-RADS 0 and CAD-RADS 1, particularly in evaluating the small low attenuation plaque and, probably, concerning the difference of expertise between the two readers.

In our series, we also evaluated the inter-observer agreement in the assessment of attenuation values within coronary lumen obtaining satisfactory results without any significant bias.

When comparing model-based and iterative reconstruction algorithms we obtained higher intra-luminal density values in each coronary vessel analyzed with MBIR compared to IR, obtaining a statistical difference for CTk, RCA, and LAD. Moreover, the absolute attenuation values obtained in our study are similar to those reported by C.H. Park et al. (12), who used a similar iodine concentration contrast agent and $80 \mathrm{kV}$ setting.

The same results were found regarding image noise: images reconstructed with MBIR reported a significantly lower noise in all vascular districts. Finally, SNR and CNR computed in MBIR images obtained a statistically significant lower noise in comparison with IR ones, according to data reported in the literature (24).

In the last ten years several prospective trials (firstly PROMISE and SCOT-HEART) and meta-analysis, evaluated the high diagnostic value and the clinical utility of coronary computed tomography proposing it as the first-line investigation in low-medium risk patients $(2,10)$. For these reasons, considering the high variability in CCTA reporting, a simple standardized scoring system for CAD classification is necessary for subsequent clinical care and to provide management recommendations (18).

The CAD-RADS is a scoring system for CCTA about vessel stenosis grade, plaque morphology, and high-risk anatomy and is based on the severity of coronary arteries stenosis with assessment from CAD-RADS 0 for the total absence of coronary plaque or stenosis to CAD-RADS 5 for complete occlusion in at least one coronary vessel. Moreover, it investigates also sub-categories as modifiers, including high-risk plaque, presence of bypass graft, or stent. The report applies to CCTA in patients with suspect or known CAD and contains recommendations to an optimal patient's management after cardiac-CT, including further testing and therapeutic options.

Several studies have proved that MBIR may simultaneously be able to reduce radiation dose and image noise with high-contrast and spatial-resolution improvement, resulting in a better assessment of the coronary artery lumen and improved visualization of plaques than IR algorithm $(21,22)$ (Fig. 3). According to these studies, our study underlines that the overall subjective image quality was better for MBIR images compared to IR ones. These results highlight the importance of

Image quality for the correct definition of CAD-RADS: the application of new reconstruction algorithms can lead to a more accurate compilation of this scoring system, in particular in the evaluation of the small vascular structure, as in the case of coronary arteries.

This study has some limitations: firstly, the study did not evaluate the impact of CAD-RADS on long-term clinical outcomes, and, secondly, there were a limited number of modifiers, which could have introduced a bias.

\section{Conclusions}


The use of a model-based reconstruction algorithm allows a significant radiation dose reduction maintaining high diagnostic image quality and reducing the overall amount of image noise. Moreover, the consistency between readers underlines the diagnostic value of low dose CCTA protocol, encouraging the use of CAD-RADS lexicon in clinical practice.

\section{Abbreviations}

ACR: American College of Radiology

ALARA: "As Low As Reasonably Achievable"

CAD-RADS: Coronary Artery Disease - Reporting And Data System

CAD: coronary artery disease

CCTA: cardiac computed tomography angiography

CM: contrast medium

CNR: contrast to noise ratio

CT: computed tomography

CTk: Common trunk

CTDI: Computed Tomography Dose Index

DLP: dose-lenght product

ED: effective dose

FBP: filtered back projection

ICA: invasive coronary angiography

LAD: Left anterior descending artery

LCx: left circumflex artery

MIP: maximum intensity projection

MPI: myocardial perfusion imaging

MPR: multiplanar reconstructions

NASCI: North American Society of Cardiovascular Imaging

NICE: National Institute for Health and Care Excellence

RCA: right coronary artery 
SCCT: Society of Cardiovascular Computed Tomography

SNR: Signal to noise ratio

VR: volume rendering

\section{Declarations}

Funding: No funding needed.

Conflicts of interest/Competing interests: Authors declared no conflict of interest.

Ethics approval: Local Ethical Committee's review of the protocol deemed that formal approval was not required owing to the retrospective, observational, and anonymous nature of this study.

Consent to participate: All patients signed the informed consent form to be eligible for this study.

Consent for publication: All patients gave consent for information about themselves to be published in scientific journals

Availability of data and material: All data generated or analyzed during this study are included in this published article.

\section{References}

1. Sun Z, Choo GH, Ng KH. Coronary CT angiography: Current status and continuing challenges. British Journal of Radiology. 2012.

2. Moss AJ, Williams MC, Newby DE, Nicol ED, Nicol ED. The Updated NICE Guidelines: Cardiac CT as the FirstLine Test for Coronary Artery Disease. Current Cardiovascular Imaging Reports, 2017,

3. Budoff MJ. The 2016 National Institute for Health and Care Excellence guidelines for chest pain: better outcomes with cardiac CT. Heart. 2018,

4. Yang L, Zhou T, Zhang R, Xu L, Peng Z, Ding J, et al. Meta-analysis: diagnostic accuracy of coronary CT angiography with prospective ECG gating based on step-and-shoot, Flash and volume modes for the detection of coronary artery disease. Eur Radiol. 2014,24(10):2345-52.

5. Samad Z, Hakeem A, Mahmood SS, Pieper K, Patel MR, Simel DL, et al. A meta-analysis and systematic review of computed tomography angiography as a diagnostic triage tool for patients with chest pain presenting to the emergency department. Journal of Nuclear Cardiology. 2012.

6. Athappan G, Habib M, Ponniah T, Jeyaseelan L. Multi-detector computerized tomography angiography for evaluation of acute chest pain - A meta-analysis and systematic review of literature. Int J Cardiol. 2010,

7. Anderson JL, Adams CD, Antman EM, Bridges CR, Califf RM, Casey DE, et al. ACC/AHA 2007 Guidelines for the Management of Patients With Unstable Angina/Non-ST-Elevation Myocardial Infarction: Executive Summary. Circulation [Internet]. 2007,116(7):803-77. Available from:

https://www.ahajournals.org/doi/10.1161/CIRCULATIONAHA.107.185752

8. Mark DB, Anderson JL, Brinker JA, Brophy JA, Casey DE, Cross RR, et al. ACC/AHA/ASE/ASNC/HRS/IAC/Mended Hearts/NASCI/RSNA/SAIP/SCAI/SCCT/SCMR/SNMMI 2014 health 
policy statement on use of noninvasive cardiovascular imaging: A report of the american college of cardiology clinical quality committee. J Am Coll Cardiol. 2014,63(7):698-721.

9. Leipsic J, Abbara S, Achenbach S, Cury R, Earls JP, Mancini GJ, et al. SCCT guidelines for the interpretation and reporting of coronary CT angiography: A report of the Society of Cardiovascular Computed Tomography Guidelines Committee. J Cardiovasc Comput Tomogr [Internet]. Elsevier, 2014 Sep 1 [cited 2018 Dec 20],8(5):342-58. Available from: https://www.sciencedirect.com/science/article/pii/S1934592514001658? via\%3Dihub

10. Cury RC, Abbara S, Achenbach S, Agatston A, Berman DS, Budoff MJ, et al. CAD-RADS ${ }^{\text {TM}: ~ C o r o n a r y ~ A r t e r y ~}$ Disease - Reporting and Data System: An Expert Consensus Document of the Society of Cardiovascular Computed Tomography (SCCT), the American College of Radiology (ACR) and the North American Society for Cardiovascular Imaging (NA. J Am Coll Radiol. 2016,

11. Xie JX, Cury RC, Leipsic J, Crim MT, Berman DS, Gransar H, et al. The Coronary Artery Disease-Reporting and Data System (CAD-RADS): Prognostic and Clinical Implications Associated With Standardized Coronary Computed Tomography Angiography Reporting. JACC Cardiovasc Imaging [Internet]. Elsevier, 2018 Jan 1 [cited 2018 Dec 20],11(1):78-89. Available from: https://www.sciencedirect.com/science/article/pii/S1936878X17309142?via\%3Dihub

12. Park $\mathrm{CH}$, Lee J, Oh C, Han KH, Kim TH. The feasibility of sub-millisievert coronary CT angiography with low tube voltage, prospective ECG gating, and a knowledge-based iterative model reconstruction algorithm. Int J Cardiovasc Imaging. Springer Netherlands, 2015,31(2):197-203.

13. Bongartz G, Golding SJ, Jurik AG, Leonardi M, Van Meerten E v. P, Geleijns J, et al. European Guidelines on Quality Criteria for Computed Tomography - EUR 16262. European Commission. 2000,1-71.

14. Deak PD, Smal Y, Kalender WA. Multisection CT Protocols: Sex- and Age-specific Conversion Factors Used to Determine Effective Dose from Dose-Length Product. Radiology. 2010,

15. Thomassin-Naggara I, Tardivon A, Chopier J. Standardized diagnosis and reporting of breast cancer. Diagn Interv Imaging [Internet]. Elsevier Masson SAS, 2014,95(7-8):759-66. Available from: http://dx.doi.org/10.1016/j.diii.2014.06.006

16. Santillan CS, Tang A, Cruite I, Shah A, Sirlin CB. Understanding LI-RADS: A primer for practical use. Magnetic Resonance Imaging Clinics of North America. 2014.

17. Tewes S, Mokov N, Hartung D, Schick V, Peters I, Schedl P, et al. Standardized reporting of prostate MRI: Comparison of the prostate imaging reporting and data system (PI-RADS) version 1 and version 2. PLoS One. 2016 ,

18. Foldyna B, Szilveszter B, Scholtz JE, Banerji D, Maurovich-Horvat P, Hoffmann U. CAD-RADS - a new clinical decision support tool for coronary computed tomography angiography. Eur Radiol. 2018,

19. Maroules CD, Hamilton-Craig C, Branch K, Lee J, Cury RC, Maurovich-Horvat P, et al. Coronary artery disease reporting and data system (CAD-RADSTM): Inter-observer agreement for assessment categories and modifiers. J Cardiovasc Comput Tomogr [Internet]. Elsevier, 2018 Mar 1 [cited 2018 Dec 20],12(2):125-30. Available from: https://www.sciencedirect.com/science/article/pii/S1934592517302551?via\%3Dihub

20. Szilveszter B, Kolossváry M, Karády J, Jermendy ÁL, Károlyi M, Panajotu A, et al. Structured reporting platform improves CAD-RADS assessment. J Cardiovasc Comput Tomogr. 2017,

21. Mehta D, Thompson R, Morton T, Dhanantwari a, Shefer E, Healthcare P. Iterative model reconstruction: simultaneously lowered computed tomography radiation dose and improved image quality. Med Phys Int. 
2013,1(2):147-55.

22. André F, Fortner P, Vembar M, Mueller D, Stiller W, Buss SJ, et al. Improved image quality with simultaneously reduced radiation exposure: Knowledge-based iterative model reconstruction algorithms for coronary CT angiography in a clinical setting. J Cardiovasc Comput Tomogr. 2017,11(3):213-20.

23. Maroules CD, Hamilton-Craig C, Branch K, Lee J, Cury RC, Maurovich-Horvat P, et al. Coronary artery disease reporting and data system (CAD-RADSTM): Inter-observer agreement for assessment categories and modifiers. J Cardiovasc Comput Tomogr. Elsevier, 2018 Mar,12(2):125-30.

24. Hirai N, Horiguchi J, Fujioka C, Kiguchi M, Yamamoto H, Matsuura N, et al. Prospective versus Retrospective ECG-gated 64-Detector Coronary CT Angiography: Assessment of Image Quality, Stenosis, and Radiation Dose. Radiology. 2008,

25. Hirai N, Horiguchi J, Fujioka C, et al. Prospective versus Retrospective ECG-gated 64-Detector Coronary CT Angiography: Assessment of Image Quality, Stenosis, and Radiation Dose. Radiology. 2008, doi: 10.1148/radiol.2482071804

\section{Figures}



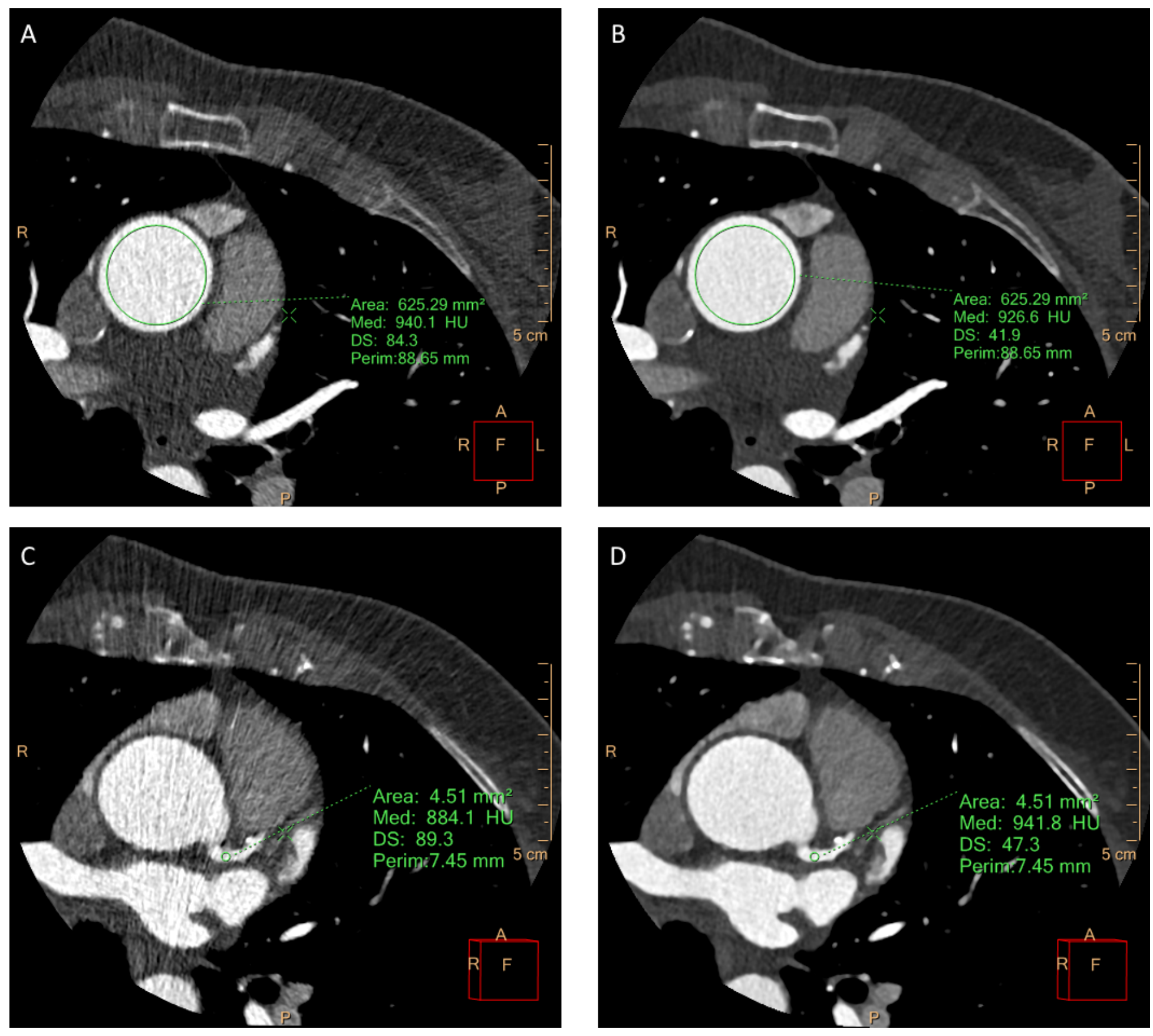

\section{Figure 1}

Evaluation of noise and $\mathrm{HU}$ of the same patient's examination dataset reconstructed with the two different iterative algorithms, model-based (MBIR) and hybrid-iterative (iDose4). CCTA was acquired with an 80-kV protocol, with a low radiation dose exposure (DLP $98.5 \mathrm{mGy}$ x cm, ED $1.44 \mathrm{mSv}$ ), with a CADARAD score of 0. A - C) Axial images reconstructed with iDose4 with circular ROI placed in the proximal ascending aorta and common trunk. B - D) Axial images reconstructed with MBIR with circular ROI placed in the same position as figure A and C. Because of the use of model-based iterative reconstruction algorithm (MBIR) we obtained an image noise reduction of $50 \%$ compared to iDose4 reconstruction as reported in the images: iDose4 standard deviation (SD) in Aorta 84 and in common trunk 89, MBIR SD: aorta 42 and common trunk 47. 
A
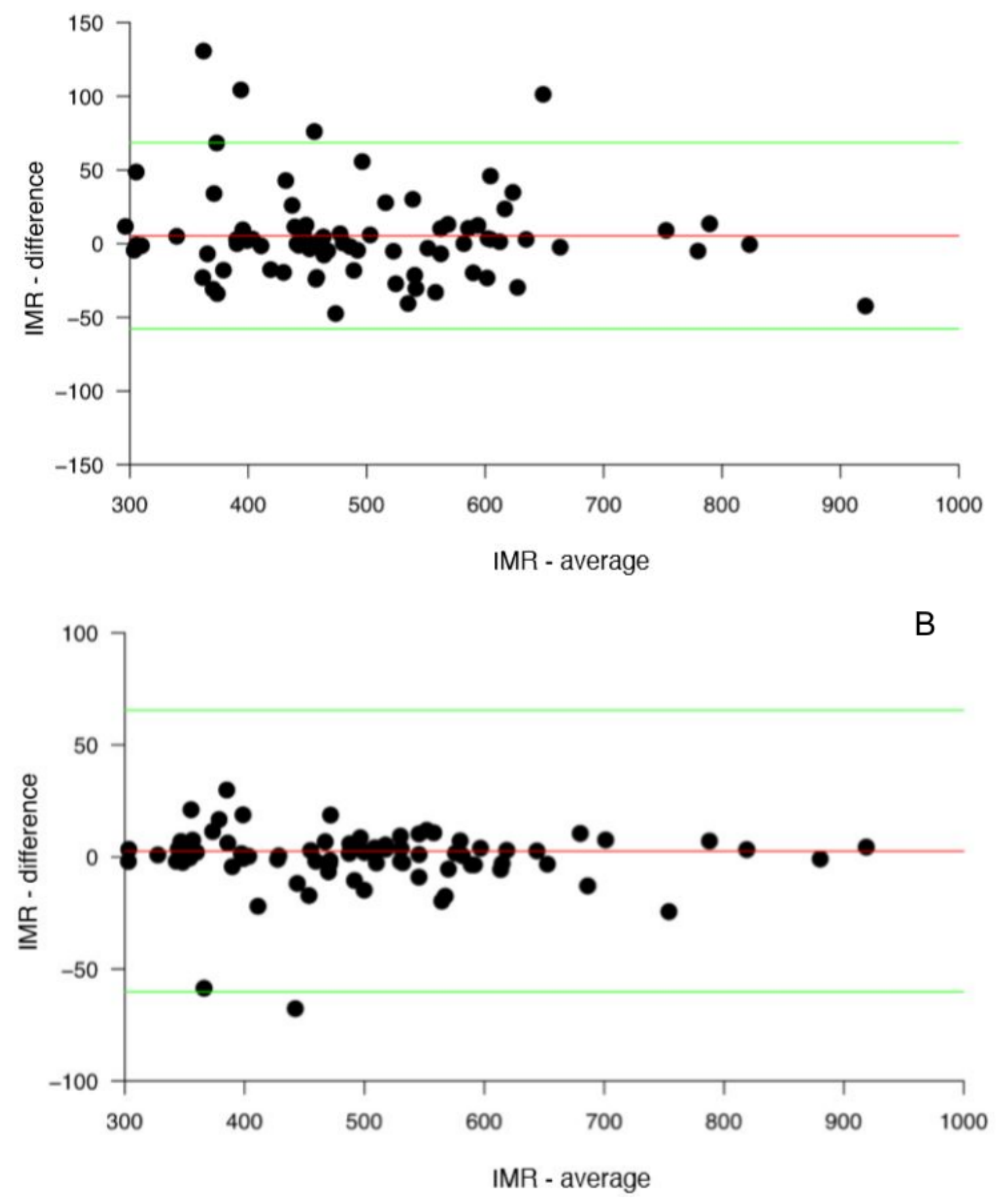

Figure 2

Comparison of Hounsfield unit (HU) with Bland-Altman analysis of right coronary artery (Fig. A) and left anterior descendant (Fig. B), between Reader 1 and Reader 2: red line shows the average of the differences and green lines show the concordance limits LoA 95\%. 

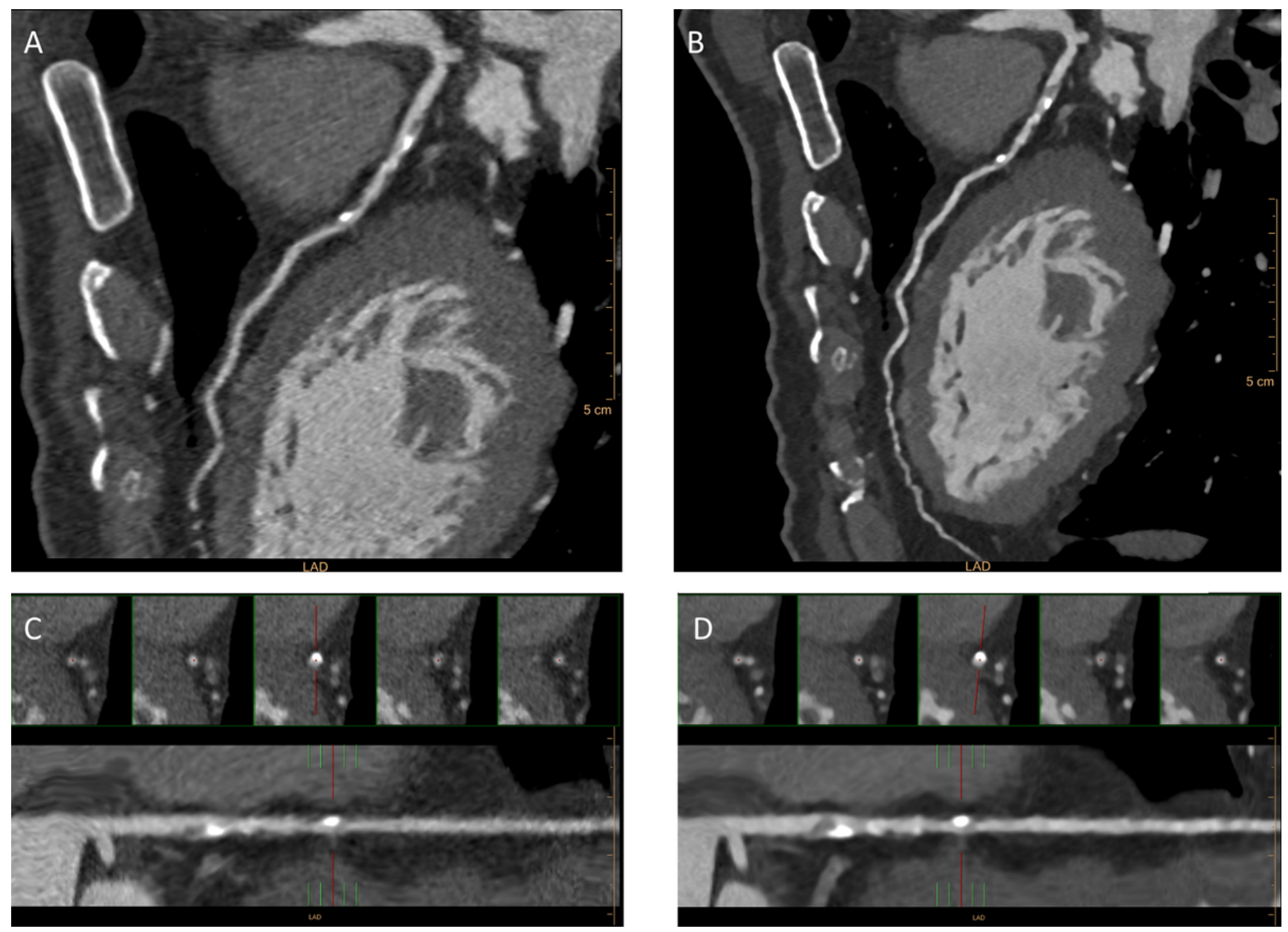

\section{Figure 3}

Evaluation of Left Anterior Descendant coronary artery of a single patient with both iDose4 (A and C) and MBIR (B and $D$ ) reconstruction algorithm. The MBIR reconstruction better depicts the artery wall and the lesions in comparison to iDose4 images because of lower image noise. A-B) Curved reconstruction of the LAD artery showing multiple mixed atherosclerotic plaques in the proximal and intermediate tract. With new model-based iterative reconstruction (MBIR) is also possible to evaluate the distal tract of the coronary artery analyzed. C-D) Straight reconstructions of iDose4 (C) and MBIR (D) images for a better evaluation of proximal atherosclerotic plaque, which determined luminal stenosis of $65-70 \%$ described as CADARDS 3. 

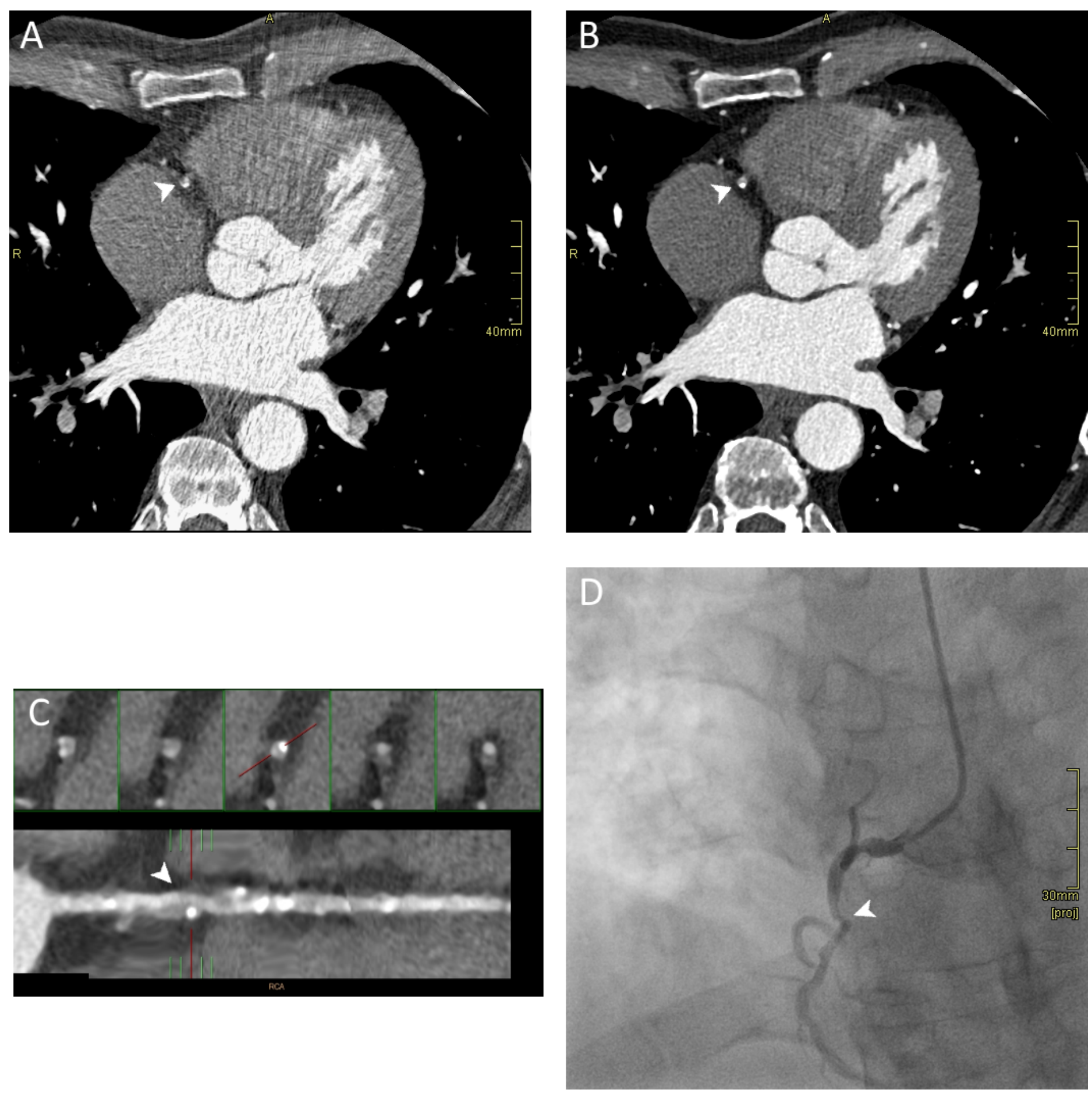

Figure 4

Comparison of CT images reconstructed with iDose4 and MBIR algorithm and Invasive Coronary Angiography (ICA) in a 65 years old male patient with thoracic pain after exercise and history of smoke and arterial hypertension. CT was acquired with 80-kV ECG-gated protocol (HR: 62 bpm). A-B) Axial image reconstructions at the same level of right coronary artery (RCA) showing the presence of fibrous and calcified atherosclerotic plaque. MBIR image presented a lower noise level and higher contrast-to-noise ratio, leading to a better evaluation of the pathological findings. C) Straight reconstruction which better shows the presence of multiple atherosclerotic plaques along the whole vessel. At the proximal tract, there is severe stenosis (arrowhead) quantified as $85 \%$ and 
reported as CADARDS 4A. D) Invasive coronary angiography showing the stenosis reported in CT examination (arrowhead), confirmed as $80 \%$. 